Q Birkhäuser 

Titu Andreescu

\section{Essential Linear Algebra with Applications}

A Problem-Solving Approach 


\author{
Titu Andreescu \\ Natural Sciences and Mathematics \\ University of Texas at Dallas \\ Richardson, TX, USA
}

ISBN 978-0-8176-4360-7

ISBN 978-0-8176-4636-3 (eBook)

DOI 10.1007/978-0-8176-4636-3

Springer New York Heidelberg Dordrecht London

Library of Congress Control Number: 2014948201

Mathematics Subject Classification (2010): 15, 12, 08

(C) Springer Science+Business Media New York 2014

This work is subject to copyright. All rights are reserved by the Publisher, whether the whole or part of the material is concerned, specifically the rights of translation, reprinting, reuse of illustrations, recitation, broadcasting, reproduction on microfilms or in any other physical way, and transmission or information storage and retrieval, electronic adaptation, computer software, or by similar or dissimilar methodology now known or hereafter developed. Exempted from this legal reservation are brief excerpts in connection with reviews or scholarly analysis or material supplied specifically for the purpose of being entered and executed on a computer system, for exclusive use by the purchaser of the work. Duplication of this publication or parts thereof is permitted only under the provisions of the Copyright Law of the Publisher's location, in its current version, and permission for use must always be obtained from Springer. Permissions for use may be obtained through RightsLink at the Copyright Clearance Center. Violations are liable to prosecution under the respective Copyright Law.

The use of general descriptive names, registered names, trademarks, service marks, etc. in this publication does not imply, even in the absence of a specific statement, that such names are exempt from the relevant protective laws and regulations and therefore free for general use.

While the advice and information in this book are believed to be true and accurate at the date of publication, neither the authors nor the editors nor the publisher can accept any legal responsibility for any errors or omissions that may be made. The publisher makes no warranty, express or implied, with respect to the material contained herein.

Printed on acid-free paper

Springer is part of Springer Science+Business Media (www.birkhauser-science.com) 


\section{Preface}

This textbook is intended for an introductory followed by an advanced course in linear algebra, with emphasis on its interactions with other topics in mathematics, such as calculus, geometry, and combinatorics. We took a straightforward path to the most important topic, linear maps between vector spaces, most of the time finite dimensional. However, since these concepts are fairly abstract and not necessarily natural at first sight, we included a few chapters with explicit examples of vector spaces such as the standard $n$-dimensional vector space over a field and spaces of matrices. We believe that it is fundamental for the student to be very familiar with these spaces before dealing with more abstract theory. In order to maximize the clarity of the concepts discussed, we included a rather lengthy chapter on $2 \times 2$ matrices and their applications, including the theory of Pell's equations. This will help the student manipulate matrices and vectors in a concrete way before delving into the abstract and very powerful approach to linear algebra through the study of vector spaces and linear maps.

The first few chapters deal with elementary properties of vectors and matrices and the basic operations that one can perform on them. A special emphasis is placed on the Gaussian Reduction algorithm and its applications. This algorithm provides efficient ways of computing some of the objects that appear naturally in abstract linear algebra such as kernels and images of linear maps, dimensions of vector spaces, and solutions to linear systems of equation. A student mastering this algorithm and its applications will therefore have a much better chance of understanding many of the key notions and results introduced in subsequent chapters.

The bulk of the book contains a comprehensive study of vector spaces and linear maps between them. We introduce and develop the necessary tools along the way, by discussing the many examples and problems proposed to the student. We offer a thorough exposition of central concepts in linear algebra through a problem-based approach. This is more challenging for the students, since they have to spend time trying to solve the proposed problems after reading and digesting the theoretical 
material. In order to assist with the comprehension of the material, we provided solutions to all problems posed in the theoretical part. On the other hand, at the end of each chapter, the student will find a rather long list of proposed problems, for which no solution is offered. This is because they are similar to the problems discussed in the theoretical part and thus should not cause difficulties to a reader who understood the theory.

We truly hope that you will have a wonderful experience in your linear algebra journey.

Richardson, TX, USA

Titu Andreescu 


\section{Contents}

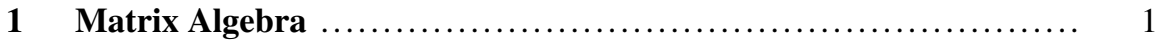

1.1 Vectors, Matrices, and Basic Operations on Them .............. 3

1.1.1 Problems for Practice............................ 10

1.2 Matrices as Linear Maps .................................. 11

1.2.1 Problems for Practice............................. 14

$1.3 \quad$ Matrix Multiplication ................................... 15

1.3.1 Problems for Practice ............................... 26

1.4 Block Matrices .......................................... 29

1.4.1 Problems for Practice.............................. 31

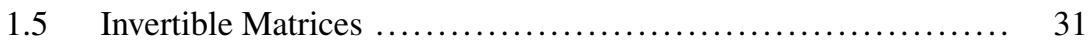

1.5.1 Problems for Practice............................. 41

1.6 The Transpose of a Matrix ............................... 44

1.6.1 Problems for Practice............................ 51

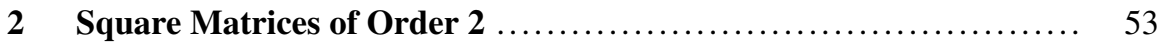

2.1 The Trace and the Determinant Maps ....................... 53

2.1.1 Problems for Practice............................ 56

2.2 The Characteristic Polynomial and the

Cayley-Hamilton Theorem ............................. 57

2.2.1 Problems for Practice........................... 65

2.3 The Powers of a Square Matrix of Order $2 \ldots \ldots \ldots \ldots \ldots \ldots \ldots \ldots . \ldots 7$

2.3.1 Problems for Practice............................. 70

$2.4 \quad$ Application to Linear Recurrences ....................... $\quad 70$

2.4.1 Problems for Practice.............................. 73

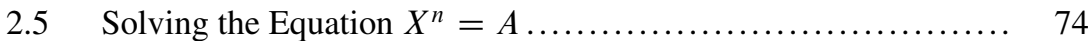

2.5.1 Problems for Practice............................ 78

2.6 Application to Pell's Equations ............................ 79

2.6.1 Problems for Practice............................. 83 


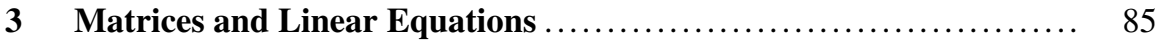

3.1 Linear Systems: The Basic Vocabulary ..................... 85

3.1.1 Problems for Practice............................ 87

3.2 The Reduced Row-Echelon form and Its

Relevance to Linear Systems .............................. 88

3.2.1 Problems for Practice............................ 95

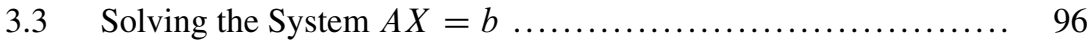

3.3.1 Problems for Practice ............................ 99

3.4 Computing the Inverse of a Matrix ......................... 100

3.4.1 Problems for Practice............................ 105

4 Vector Spaces and Subspaces................................ 107

4.1 Vector Spaces-Definition, Basic Properties and Examples ........ 107

4.1.1 Problems for Practice.............................. 113

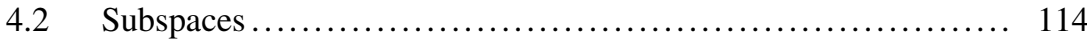

4.2.1 Problems for Practice............................... 121

4.3 Linear Combinations and Span ........................... 122

4.3.1 Problems for Practice ............................ 127

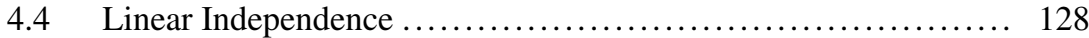

4.4.1 Problems for Practice ........................... 133

4.5 Dimension Theory.................................... 135

4.5.1 Problems for Practice............................. 146

$5 \quad$ Linear Transformations.................................... 149

5.1 Definitions and Objects Canonically Attached to a Linear Map ... 149

5.1.1 Problems for practice .............................. 157

5.2 Linear Maps and Linearly Independent Sets .................. 159

5.2.1 Problems for practice ........................... 163

5.3 Matrix Representation of Linear Transformations ............... 164

5.3.1 Problems for practice ............................. 181

5.4 Rank of a Linear Map and Rank of a Matrix.................. 183

5.4.1 Problems for practice ............................ 194

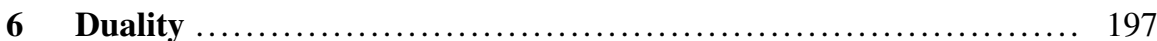

6.1 The Dual Basis ........................................ 197

6.1.1 Problems for Practice............................... 208

6.2 Orthogonality and Equations for Subspaces ................. 210

6.2.1 Problems for Practice............................. 218

6.3 The Transpose of a Linear Transformation .................. 220

6.3.1 Problems for Practice.............................. 224

6.4 Application to the Classification of Nilpotent Matrices .......... 225

6.4.1 Problems for Practice............................ 234

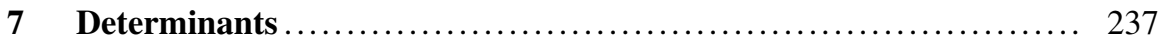

$7.1 \quad$ Multilinear Maps ..................................... 238

7.1.1 Problems for Practice .......................... 242 
7.2 Determinant of a Family of Vectors, of a Matrix, and of a Linear Transformation.

7.2.1 Problems for Practice............................. 251

7.3 Main Properties of the Determinant of a Matrix ............... 253

7.3.1 Problems for Practice............................ 262

7.4 Computing Determinants in Practice......................... 264

7.4.1 Problems for Practice.............................. 278

7.5 The Vandermonde Determinant ........................... 282

7.5.1 Problems for Practice.......................... 287

7.6 Linear Systems and Determinants ....................... 288

7.6.1 Problems for Practice......................... 298

8 Polynomial Expressions of Linear Transformations and Matrices ... 301

8.1 Some Basic Constructions ................................. 301

8.1.1 Problems for Practice........................... 303

8.2 The Minimal Polynomial of a Linear Transformation or Matrix .. 304

8.2.1 Problems for Practice............................. 309

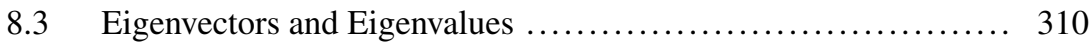

8.3.1 Problems for Practice.............................. 316

8.4 The Characteristic Polynomial.............................. 319

8.4.1 Problems for Practice ............................ 330

8.5 The Cayley-Hamilton Theorem .......................... 333

8.5.1 Problems for Practice......................... 337

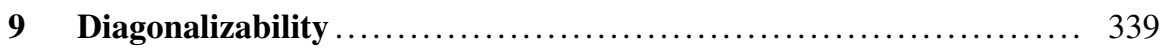

9.1 Upper-Triangular Matrices, Once Again ..................... 340

9.1.1 Problems for Practice.......................... 343

9.2 Diagonalizable Matrices and Linear Transformations........... 345

9.2.1 Problems for Practice........................... 356

9.3 Some Applications of the Previous Ideas .................... 359

9.3.1 Problems for Practice.......................... 372

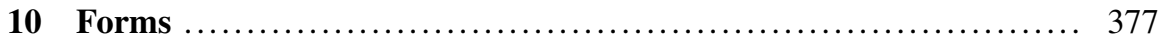

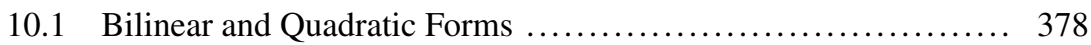

10.1.1 Problems for Practice.......................... 389

10.2 Positivity, Inner Products, and the Cauchy-Schwarz Inequality ... 391

10.2.1 Practice Problems................................... 397

10.3 Bilinear Forms and Matrices ............................... 399

10.3.1 Problems for Practice............................... 406

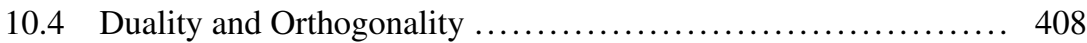

10.4.1 Problems for Practice.............................. 416

10.5 Orthogonal Bases......................................... 418

10.5.1 Problems for Practice ........................... 436

10.6 The Adjoint of a Linear Transformation .................... 442

10.6.1 Problems for Practice............................. 448

10.7 The Orthogonal Group ................................. 450

10.7.1 Problems for Practice........................ 465 
10.8 The Spectral Theorem for Symmetric Linear

Transformations and Matrices ............................ 469

10.8.1 Problems for Practice........................ 477

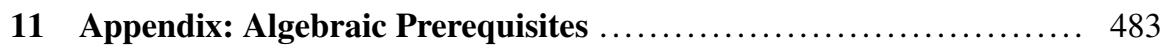

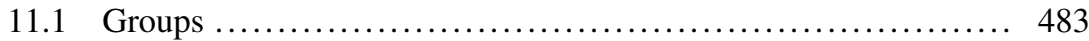

11.2 Permutations.............................................. 484

11.2.1 The Symmetric Group $S_{n} \ldots \ldots \ldots \ldots \ldots \ldots \ldots \ldots \ldots . \ldots \ldots 4$

11.2.2 Transpositions as Generators of $S_{n} \ldots \ldots \ldots \ldots \ldots \ldots \ldots . \ldots 46$

11.2.3 The Signature Homomorphism .................. 487

11.3 Polynomials ..................................... 489

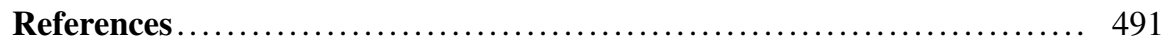

